\title{
loT-based detection and support technology of surrounding rock in high-stress region
}

\author{
Luo Sun
}

\author{
Beijing Polytechnic College, Beijing, 100042, China
}

\begin{abstract}
Keywords: IoT, highland, stress area, surrounding rock, exploration, support, technology
\end{abstract}
\begin{abstract}
In order to obtain the stability and stress data of the surrounding rock mass in the deep region of the mine, a reasonable support scheme and support parameters are adopted to reduce the degree of roadway deformation and damage in the deep region and reduce the cost of roadway maintenance. There are local weak and weak tectonic belts such as cracks and also joints in the surrounding rock in the deep area of the mine. At the same time, after the excavation, cracks and fracture zones will be then further generated, affecting the continuous stability of the roadway. It is necessary to detect and control the structure and damage of surrounding rock so as to adopt targeted support schemes.
\end{abstract}

\section{Introduction}

Pingmei Coal No. 1 Mine is an old coal mine located in the central mining area. The depth of the mining is $1075 \mathrm{~m}$. The surrounding rocks in deep rock lanes are dominated by sandy mudstones and mudstones. They are easily deformed due to large burial depth and high ground stress and cause roadway deformation with destruction, thus affecting safety production.

In order to obtain the stability and stress data of the surrounding rock mass in the deep region of the mine, a reasonable support scheme and support parameters are adopted to reduce the degree of roadway deformation and damage in the deep region and reduce the cost of roadway maintenance. In the deep mine area of the mine, the No. 1 mine conducted panoramic digital drilling analysis, flow stress recovery method test ground stress, and deep-rock deep rock surrounding rock loosening ring test experiments. The support plan was adopted to improve the support effect of deep soft rock roadway.

The concept of the Internet of Things was put forward in 1999. The Internet of Things is based on the Internet, RFID technology, and EPC standards. Based on the computer Internet, using radio frequency identification technology and wireless data communication technology, a real-world internet "Internet of things" that enables real-time sharing of global item information is constructed. Networking) Although there is not yet a unified standard definition for the Internet of Things at present in China, from the perspective of the Internet of Things, the Internet of Things is a kind of convergent application and technology upgrade that appears after a certain period of development of modern information technology. Perceiving technologies, modern network technologies, and the integration and integration of artificial intelligence and automation technologies enable people and objects to have a smart dialogue and create a smart world. Because the development of Internet of Things technology involves almost every aspect of information technology, it is a kind of convergent and systematic innovation application and development, and therefore it is only known as the third revolutionary innovation of the information industry.

The essence of the Internet of Things is summarized in three main areas:

1. The characteristics of the Internet, that is, the Internet that must be able to interconnect and interoperate with objects that require networking;

2. Identification and communication features, that is, the "things" incorporated into the Internet of Things must have the functions of automatic identification and object-based communication (M2M);

3. Intelligent features, that is, network systems should have the characteristics of automation, self-feedback and intelligent control. 


\section{The Proposed Methodology}

IoT. The implementation of IoT in practical applications requires the participation of all walks of life. It also requires the guidance of national governments and related laws and policies. The development of the Internet of Things has scale, extensive participation, management, technical, and material properties. Such characteristics, among them, technical issues are the most critical issues of the Internet of Things. The Internet of Things technology is a comprehensive technology and is a system.

In general, the steps for the development of the Internet of Things are as follows:

1. Identify the properties of the object. Properties include static and dynamic properties. Static properties can be stored directly in the tag. Dynamic properties need to be detected by the sensor in real time.

2. The need to identify the device to complete the reading of the object properties, and convert the information into a data format suitable for network transmission;

3. The information of the object is transmitted to the information processing center through the network, and the processing center completes the related calculation of the object communication.

Of course, there are two important factors to really establishing an effective Internet of Things:

1. Scale, only if it has a scale, can the intelligence of the goods plays a role;

2. Liquidity: Items are not always stationary, but they are in a state of movement. It is necessary to keep the articles in motion, and even at high speeds, the dialogue can be achieved at any time.

In the Internet of Things, radio frequency identification technology is a very important technology. In a radio frequency identification system, a tag may be embedded in any item in advance, such as people's daily life items, but due to the owner of the item (such as clothing), it may not necessarily be able to perceive that the item is pre-embedded with an electronic tag and itself. It may be scanned, located, and tracked in an uncontrolled manner, which inevitably infringes on one's privacy. Therefore, how to ensure that the privacy of the owner of the label is not infringed is a key issue for radio frequency identification technology and the promotion of the Internet of Things. Moreover, this is not only a technical issue but also involves political and legal issues. This issue must be highly regarded and solved technically and legally. The key to infringing privacy concerns lies in the basic function of RFID tags: the identification (ID) or identification code of any tag can be arbitrarily scanned remotely, and the tag automatically responds to the reader's instructions indiscriminately. Transfers its stored information to the reader. This feature can be used to track and locate a specific user or item to obtain relevant private information. This raises the question of how to ensure that the privacy of the owner of the embedded tagged item is not infringed.

The current sensing technology of the Internet of Things is mainly RFID. The products embedded in this chip are likely to be perceived by anyone. It is a system for the owner of the product that can be easily managed. However, it also has a huge problem. Other people can also perceive it, such as the product's competitors. How can we use this valuable information in the process of perception, transmission, and application? Used by others, it is not used by competitors. This requires a lot of work on security and a strong security system. It should now be said that what security problems will arise, how to deal with these security issues, and how to shield them are all very complicated issues, and even unclear. But these issues must be worth noting, especially the provider of this management platform.

The design, construction and operation of the IoT industry applications. Developers and operators at the application layer, select the required resources and functions (including information technology and industry technology), through the call to the underlying services to build their own industry applications. In the IoT technology-level system, the five layers below the application layer can all be understood as the "hardware" of the application layer; "software" is the application logic written by the application developer itself.

If the application software is hosted on SAAS (software as a service) and the underlying system is provided by a cloud computing service provider, then the "hardware" of any physical entity may not be visible in the application. Instead, it is the lower level modularization and standardization. Information services and industry functions. 
Surrounding Rock Drilling Panoramic Observation and Analysis Technology in Roadway. There are local fractures, joints and other weak structural belts in the surrounding rock in the deep region of the mine. At the same time, cracks and fracture zones will be further generated after the excavation, which will affect the continuous stability of the roadway. It is necessary to detect and control the surrounding rock structure and damage, so as to take targeted Support program. The traditional rock wall structure detection technology mainly adopts the borehole spectrometer, which can intuitively observe the borehole on the roadway wall. The disadvantage is that it cannot realize the digital splicing, fracture surface recognition and quantitative description. Therefore, the digital panoramic borehole observation and analysis technology developed by the Wuhan Institute of Rock and Soil Mechanics, Chinese Academy of Sciences was used to observe and analyze the boreholes so as to grasp the geological conditions of the surrounding rocks and provide basis for the design.

The test sites were selected to be at the three levels of one mine and were to be lowered by the Wuyi-1 track. The depth of the experimental roadway was $880 \mathrm{~m}$ deep, the roadway and the drill hole were located near the top of the coal seam, and the wall rocks were dominated by sandy mudstone and mudstone. From the analysis of geological data and borehole data near the coal seam, there are Zhangjia reverse faults and normal faults in the southwest corner of the mining area. These two faults are all oriented in the northwest direction. The Zhangjia reverse fault passes through the mining area, and normal faults are extracted from the mining area. The area is not far from extending, and there will be more secondary small faults in this area.

According to the geology of the site, in order to increase the accuracy of the calculation, the mesh of the area where the roadway is located is densely distributed, and the mesh of other areas is coarsened. The model length $\times$ width $\times$ height $=55 \mathrm{~m} \times 50 \mathrm{~m} \times 44 \mathrm{~m}$, block 520000 blocks, node 543090, simulated roadway width $5000 \mathrm{~mm}$, height $4000 \mathrm{~mm}$, and the grid around the roadway represents $0.5 \mathrm{~mm}$. The depth of the floor is about $800 \mathrm{~m}$. The upper boundary of the model is the self-gravity of the overlying rock mass, about $20 \mathrm{MPa}$, and the horizontally applied stress is calculated with a pressure coefficient of 1.0 to limit the displacement and velocity in the $\mathrm{x}, \mathrm{y}, \mathrm{and} \mathrm{z}$ directions. For the coal rock mass, which is a geological model of the characteristics of high in-situ stress rock, the Coulomb-Mohr criterion is adopted.

When the support strength was increased from $0.05 \mathrm{MPa}$ to $0.3 \mathrm{MPa}$, the roof subsidence decreased from $225 \mathrm{~mm}$ to $157 \mathrm{~mm}$; the deformation of the floor was maintained at approximately $450 \mathrm{~mm}$. When the supporting strength increases from $0.3 \mathrm{MPa}$ to $0.4 \mathrm{MPa}$, the amount of roof subsidence decreases sharply to $54.9 \mathrm{~mm}$; the amount of bottom plate deformation rapidly decreases to $118 \mathrm{~mm}$. The simulation results show that when the support strength is less than $0.3 \mathrm{MPa}$, the roof subsidence is maintained at about $200 \mathrm{~mm}$. Affected by many factors, the actual amount of subsidence is generally greater than the simulated value. The deformation of the bottom plate is 450 $\mathrm{mm}$, which is about 2 times of the deformation of the top plate, indicating that the bottom drum phenomenon is obvious and is close to the actual measurement of the roadway.

Left-hand displacement is slightly larger than right-hand. When the support strength increased from 0.05 MPa to 0.3 MPa, the single-handed approach amount had a small decrease. When the supporting strength continues to increase, the two groups of approaching amount decreases, the decreasing extent increases, and when the supporting strength increases from $0.3 \mathrm{MPa}$ to $0.4 \mathrm{MPa}$, the displacement of the two gangs decreases by approximately $115 \mathrm{~mm}$.

When the support strength is $0.05 \mathrm{MPa}$, the peak value of vertical stress reaches $1.67 \mathrm{MPa}$. With the increase of support strength, the peak value of vertical stress tends to decrease. When the support strength reaches $0.4 \mathrm{MPa}$, the peak value of vertical stress is $14.3 \mathrm{MPa}$. The simulation results show that as the support strength increases, the vertical stress peak moves toward the shallow rock, but the moving range is small. In addition, when the support strength is greater than $0.3 \mathrm{MPa}$, the support strength is further increased, and the magnitude of the vertical stress peak and the position of the roadway are almost unchanged.

With the increase of supporting strength, the initial stage of horizontal stress remains almost unchanged and gradually decreases in the subsequent stage. When the supporting strength is 0.05 $\mathrm{MPa}$, the horizontal stress peak value reaches $15.8 \mathrm{MPa}$; when the supporting strength increases to 
0.4 MPa, the level The peak stress drops to $15 \mathrm{MPa}$. The simulation results show that with the increase of supporting strength, the peak stress of the roof moves to the shallow part of the surrounding rock, and the moving range is small. The peak stress of the floor level moves to the deep of the surrounding rock due to its fracture.

Support effect monitoring. In order to verify the supporting effect, the monitoring of the deep base point displacement of the roof was conducted in the test roadway. Three base stations are arranged on the floor to perform deep base point displacement monitoring. The monitoring results are as follows.

In the early stage of excavation, the amount of roof subsidence was large and the deformation rate was rapid, and then the amount of subsidence increased slowly. The monitoring shows that the shallow part of the roof is broken within $0 \sim 1 \mathrm{~m}$, and the $3 \sim 5 \mathrm{~m}$ layer is relatively large. According to the monitoring data, it can be known that: (1) In the first 10 to 20 days before tunnel excavation, the displacement increases rapidly, the amount of roof subsidence is generally $150 \sim 200 \mathrm{~mm}$, and the two groups approach to 100 150 mm; (2) After 20 days, the displacement increased slowly, and the roof subsidence and the two groups of displacements changed little; (3) After 30 to 40 days, the roof subsidence and the two subsidences tend to be stable, with the roof subsidence. Stable at 200 250 $\mathrm{mm}$, and the two groups approaching the stability of about $200 \mathrm{~mm}$, which shows that the roof of the $5 \mathrm{~m}$ long anchor rod played a supporting role, and can well control the roof, the use of the Ministry 3.7 The $\mathrm{m}$ bolt also effectively controls the relative proximity of the two gangs.

\section{Conclusion}

Through the use of borehole panoramic digitization detection and analysis techniques in the floor and the bulge of deep rock lanes, the surrounding rock structure was detected and analyzed, and the stress of the surrounding rock was tested using the flow stress recovery method for a long period of time. The in-situ stress data were obtained using the cross the hole acoustic wave method was used to test the condition of loose rock around the surrounding rock. The surrounding rock and stress distribution characteristics were obtained, and the failure mechanism of the surrounding rock was explored. At the site, anchor network cable spray + wall post injection + combination anchor cable grouting support method was adopted to ensure the deep roadway support effect, reduce the maintenance cost of the support roadway, and ensure the safety production.

\section{References}

1. León, J. C. B. D., Cortes, D. A. P., Martínez, A. R., \& Bedoya, J. W. B. (2017). Computational Detection of Salient Information to Identify High Stress and Ambiguity Regions in Digital Photoelasticity Images. Imaging Systems and Applications (pp.IM4E.2).

2. Onyemaobi, I., Ayalew, H., Liu, H., Khm, S., \& Yan, G. (2018). Identification and validation of a major chromosome region for high grain number per spike under meiotic stage water stress in wheat (triticum aestivum l.). Plos One, 13(3), e0194075.

3. Wang, Y., Li, J., \& Wang, H. H. (2017). Cluster and cloud computing framework for scientific metrology in flow control. Cluster Computing(1), 1-10.

4. Giri, A., Bharti, V. K., Vivek, P., \& Kalia, S. (2017). Health and productivity of dairy cattle in high altitude cold desert environment of leh-ladakh: a review. Indian Journal of Animal Sciences, 87(1), 3-10.

5. Qiu, J., Lei, D., Hu, J., Yin, T., Zhang, K., \& Yu, D., et al. (2017). Effect of intraplaque angiogenesis to atherosclerotic rupture-prone plaque induced by high shear stress in rabbit model. Regenerative Biomaterials, 4(4), 215-222.

6. Cai, Z., Deng, L., Li, D., Yao, X., Cox, D., \& Wang, H. (2017). A fcm cluster: cloud networking model for intelligent transportation in the city of macau. Cluster Computing(3), 1-10. 
7. Mohamad, M., Soin, N., \& Ibrahim, F. (2017). Design of a high sensitivity MEMS piezoresistive intracranial pressure sensor using three turns meander shaped piezoresistors. International Conference on Bio-Engineering for Smart Technologies (pp.1-4). IEEE. 